\title{
Ultra Reliable Communication via CC-HARQ in Finite Block-Length
}

\author{
Endrit Dosti, Mohammad Shehab, Hirley Alves and Matti Latva-aho \\ Centre for Wireless Communications (CWC), University of Oulu, 90014 Oulu, Finland \\ email:firstname.lastname@oulu.fi
}

\begin{abstract}
In this paper, we suggest a power allocation strategy for the Chase Combining Hybrid Automatic Repeat Request (CCHARQ) protocol with ultra-reliability constraints. The proposed optimal power allocation scheme would allow us to reach any outage probability target in the finite block-length regime. We cast an optimization problem as minimization of the average transmitted power under a given outage probability and maximum transmit power constraint. To solve the problem and attain the closed form solution, we utilize the Karush-Kuhn-Tucker (KKT) conditions. We show that in the finite block-length regime the transmitted power is highly dependent on the number of channel uses. However, as the block size increases, the transmitted power becomes constant. Furthermore, we show that by using the proposed power allocation scheme, we can achieve very large average and sum power gains when compared to the one shot transmission.
\end{abstract}

\section{INTRODUCTION}

Mobile communication systems play a very important role in everyone's daily life. Historically, with each generation of mobile systems, there has been a trend to provide higher data rates. This is expected to continue in the future generations of mobile systems. However, new features will be introduced in order to improve the user experience [1]. The vision is to connect all devices that benefit from an internet connection [2]. This will result in the creation of the Internet of Things (IoT). A key characteristic of the IoT is that most of the wireless connections are expected to be generated by the autonomous devices. Therefore, the implementation of this vision requires wireless systems which are able to support very large number of devices simultaneously, and at the same time fulfill very stringent requirements on delay and reliability [3].

In the fifth generation of mobile communication systems $(5 \mathrm{G})$, at least two new operation modes will be introduced: $(i)$ massive Machine type communication (m-MTC), which aims at providing connectivity for large number of low-rate and lowpower devices (e.g. sensors or actuators) [4]; and (ii) Ultra Reliable Communications, which targets ultra-reliable, low latency communication [5]. Therein, we require the communication link to have an outage probability much lower than $0.0001 \%$ and tactile latency lower than $1 \mathrm{~ms}$ [6]. Generally, most of the devices in a MTC network will be rather simple. Therefore, the amount of information they transmit will be small. Despite its size, the transmitted information needs to be decoded at the intended receiver with very high reliability and very low latency.

One major issue in wireless communications is the presence of fading, which causes fluctuations in the received signal power, thus resulting in loss of transmitted packets [7]. To alleviate this effect, retransmission schemes can be utilized. Therein, we transmit the same packet in several independent fading paths and then combine them accordingly at the receiver. In this context, Chase Combining Hybrid Automatic Repeat Request (CC-HARQ) protocol significantly improves the reliability level while maintaining certain complexity [8]. Thus, the probability of a successful transmission in the wireless communication link is increased by retransmitting the packets that experienced bad channel conditions.

In this paper, to meet the requirements of URC, we resort to finite block-length coding. It allows us to reduce the size of the messages, therefore latency, while guaranteing reliability. In finite block-length, the length of metadata (control information) is of comparable size to the actual data, thus classical performance metrics (e.g. channel capacity or outage capacity) become highly suboptimal [9]. Thus, it would be of high interest to evaluate the performance of retransmission schemes with optimal power allocation. Little work has been done in this field for the short packets domain. For instance, in [10] the authors propose an optimal power allocation strategy for type-I ARQ protocol, which achieves a target outage probability for a fixed maximum number of transmissions. However, the protocol that is introduced in the paper is quite simplistic and does not provide any combining gains. Further, in [11] the performance of incremental redundancy ARQ is evaluated for aditive white Gaussian noise (AWGN) channel. The authors show that a large number of retransmissions enhances performance in terms of the long term average transmission rate (LATR). This scheme requires a more sophisticated encoding/decoding mechanism that may not be feasible in nodes with limited computational capabilities. Moreover, authors do not assess the impact of such large number of retransmissions on latency nor do they guarantee high reliability. Further, in [12] the authors analyze the performance of ARQ protocol over the fading channel under the assumption of infinite number of transmissions and an instantaneous and error free feedback. However, they do not focus on the impact of power allocation between different ARQ rounds. A power allocation scheme that minimizes the outage probability is developed in [13], but only for the case of two transmissions. Their proposed algorithm does not guarantee a minimal outage probability level, which would be essential in the case of URC. 
In this paper, we develop a power allocation scheme for CC-HARQ protocol that allows us to achieve any target reliability level in block-fading channels. We analyze the performance for the general case of $M$ transmissions and we provide closed form expressions for the transmission power of each CC-HARQ round. Furthermore, we show that the proposed scheme enables large gains, in terms of both average and maximum power, when compared to the one shot transmission approach.

\section{SYSTEM MODEL}

Assume a transmitter-receiver pair communicating via CC-HARQ protocol, where the maximum number of transmission rounds is set to $M$. In each round, a scaled version of the initial codeword will be transmitted. When this protocol is implemented, the receiver buffers the packets and performs maximal ratio combining to enhance the SNR. Once the maximum number of transmissions are exhausted, the packets are discarded and error is declared. The operating principle of the protocol is illustrated in Fig. 1.

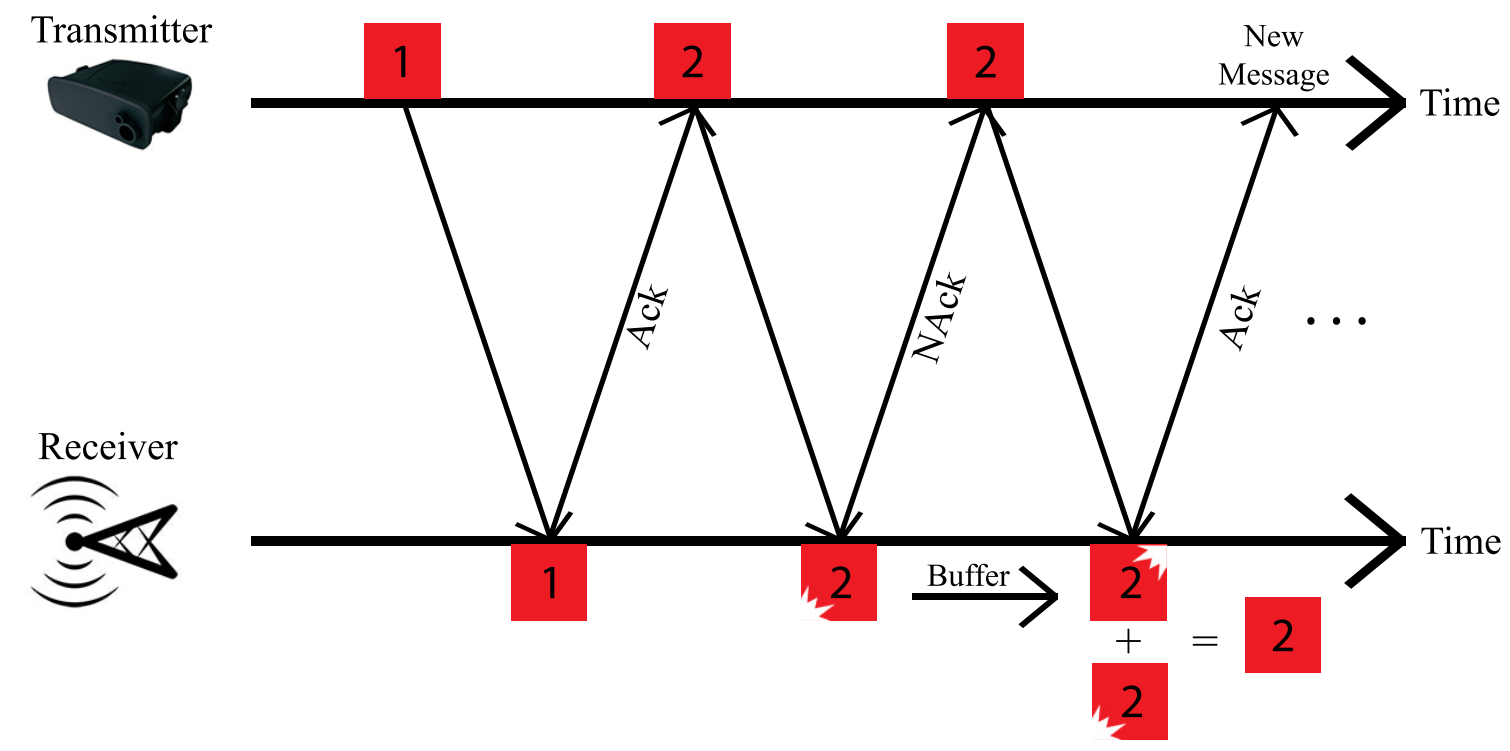

Fig. 1. The setup for CC-HARQ protocol. The transmitter sends first packet 1 and waits for the acknowledgment from the receiver. Then, he sends packet 2 . If the receiver can not decode the packet, it buffers the packet and asks for retransmission. Afterwards, when it receives packet 2 once more, it combines it with the buffered packet to extract the information.

Consider quasi-static Rayleigh fading channel conditions, in which the channel gain $h \sim \mathcal{C N}(0,1)$ remains constant for the duration of one packet transmission and changes independently between CC-HARQ rounds. We assume that the receiver has channel state information while the transmitter knows only the distribution of the channel gains and the information it obtains from the feedback. Then the received signal at the $m^{\text {th }}$ round can be written as

$$
y_{m}=\sqrt{\rho_{m}} h_{m} x_{m}+w_{m},
$$

where $x_{m}$ is the transmitted signal and $w_{m}$ is the AWGN noise term with noise power $N_{0}=1$. The term $\rho_{m}$ is the packet transmit power, which since the variance of the noise is set to 1, corresponds to the transmission signal-to-noise ratio (SNR).

\section{MAXIMUM CODING RATE IN FINITE BLOCK-LENGTH}

In this section, we briefly summarize the recent results in the characterization of the maximum channel coding rate and outage probability in the finite block-length regime. Further, we discuss the scenario of the one shot transmission.

For notational convenience we need to define an $(n, K, \rho, \epsilon)$ code as a collection of

- An encoder $\mathcal{F}:\{1, \ldots, K\} \mapsto \mathcal{C}^{n}$ which maps the message $k \in\{1, \ldots, K\}$ into an $n$-length codeword $c_{i} \in\left\{c_{1}, \ldots, c_{n}\right\}$ such that the following power constraint $(\rho)$ is satisfied:

$$
\frac{1}{n}\left\|c_{i}\right\|^{2} \leqslant \rho, \forall i .
$$


- A decoder $\mathcal{G}: \mathcal{C}^{n} \mapsto\{1, \ldots, K\}$ that satisfies the maximum error probability $(\epsilon)$ constraint:

$$
\max _{\forall i} \operatorname{Pr}[\mathcal{G}(y) \neq I \mid I=i] \leqslant \epsilon,
$$

where $y$ is the channel output induced by the transmitted codeword according to (1).

The maximum achievable rate of the code is defined as:

$$
R_{\text {max }}^{*}(n, \rho, \epsilon)=\sup \left\{\frac{\log K}{n}: \exists(n, K, \rho, \epsilon) \text { code }\right\},
$$

where $\log$ refers to the natural logarithm.

For the AWGN channel non-asymptotic lower and upper bounds on the maximum achievable rate have been derived in [14]. Recently, a tight approximation for $R_{\max }^{*}(n, \rho, \epsilon)$ has been proposed for sufficiently large values of $n$ in the case of the quasi-static fading channel [15] and is given by

$$
R_{\text {max }}^{*}(n, \rho, \epsilon) \approx C_{\epsilon}+\mathrm{O}\left(\frac{\log n}{n}\right),
$$

where $C_{\epsilon}$ is the outage capacity:

$$
C_{\epsilon}=\sup \{R: \operatorname{Pr}[\log (1+\rho \cdot z)<R]<\epsilon\} .
$$

In [10], the authors have shown that in the ultra reliable regime $\left(\epsilon<10^{-3}\right)$, an asymptotic approximation can be used. In this regime, the maximum achievable rate (5) converges to the one with asymptotically long codewords $R^{*}(n, \epsilon)=C_{\epsilon}$, where $C_{\epsilon}$ is defined in (6). Therefore, when power is high enough, the outage probability in the $m^{t h}$ round can be calculated as:

$$
\epsilon_{m}=\operatorname{Pr}[\log (1+\rho \cdot z)<R]=1-e^{\frac{e^{R}-1}{\rho_{m}}} \approx \frac{\phi}{\rho_{m}},
$$

where $\phi=e^{R}-1$. The equality in (7) holds for Rayleigh fading channels. The approximation in (7) is obtained via the first order of Taylor expansion $e^{-x} \approx 1-x$.

For ultra reliable communications, we are required to achieve very low outage probabilities $\epsilon$, while consuming minimal power. However, achieving this task via single shot transmission can be a cumbersome task [10], [13]. Thus, we resort to utilization of retransmission schemes. Specifically, to achieve the target outage probability, we focus on the CC-HARQ protocol with optimal power allocation.

\section{Power ALlocation}

In this section, we evaluate the impact of the CC-HARQ protocol on the outage probability of the user's transmission. For this purpose, we cast an optimization problem as a minimization of the average transmission power subject to an outage probability constraint, as well as non-negative power terms. By solving the problem, we propose an optimal power allocation scheme for the CC-HARQ protocol that allows us to reach any target outage probability assuming that we can have up to $M$-transmissions.

The main issue is to meet the outage probability requirements, while spending as little power as possible for conveying the information. Since, when utilizing CC-HARQ protocol we will have multiple transmissions, a conventional approach would be to allocate equal power in each round. This implies that, given a certain power budget $\rho_{b u d g e t}$, the transmit power in the $m^{\text {th }}$ transmission round would be $\rho_{m}=\frac{\rho_{\text {budget }}}{M}$. However, such simplistic approach is shown to be highly suboptimal for very low outage probability values [13], [16]. Thus, to achieve our target outage probability, we propose a power allocation algorithm in order to minimize the average transmitted power. Bearing this in mind, the average transmitted power can be defined as

$$
\rho_{\text {avg }}=\frac{1}{M} \sum_{m=1}^{M} \rho_{m} E_{m-1},
$$

where $M$ is the maximum number of CC-HARQ rounds, $\rho_{m}$ is the power transmitted in the $m^{\text {th }}$ round and $E_{m-1}$ is the outage probability up to the $m-1$ round. Next, we compute the probability that the packet can not be decoded correctly even after the maximum allowed number of retransmissions. It corresponds to the outage probability up to the $M^{\text {th }}$ round $\left(E_{M}\right)$. We can obtain a very tight approximation of $E_{M}$ for the CC-HARQ protocol as

$$
E_{M}=\frac{\phi^{M}}{M ! \prod_{m=1}^{M} \rho_{m}} .
$$

Notice that (9) is obtained by making some minor improvements of the approximation presented in [17]. Further, let the outage probability up to the first transmission be $E_{0}=1$. 


$$
\begin{aligned}
\frac{\partial \mathcal{L}\left(\rho_{m}, \mu_{m}, \lambda\right)}{\partial \rho_{m}} & =\frac{1}{M} \frac{\phi^{m-1}}{(m-1) ! \prod_{i=1}^{m-1} \rho_{i}}-\lambda\left(\frac{\phi^{M}}{M ! \rho_{m}^{2} \prod_{i=1, i \neq m}^{M} \rho_{i}}\right)-\frac{1}{M} \sum_{i=1}^{M-m} \frac{\rho_{m+i} \phi^{m+i-1}}{(m+i-1) ! \rho_{m}^{2} \prod_{j=1, j \neq m}^{m+i-1} \rho_{j}}-\mu_{m} . \\
\frac{\partial \mathcal{L}\left(\rho_{m}, \mu_{m}, \lambda\right)}{\partial \rho_{M}} & =\frac{\phi^{M-1}}{M ! \prod_{i=1}^{M-1} \rho_{i}}-\lambda\left(\frac{\phi^{M}}{\rho_{M}^{2} M ! \prod_{i=1, i \neq m}^{M} \rho_{m}}\right)=0 . \\
\frac{\partial \mathcal{L}\left(\rho_{m}, \mu_{m}, \lambda\right)}{\partial \rho_{M-1}} & =\frac{\phi^{M-2}}{M(M-2) ! \prod_{i=1}^{M-2} \rho_{i}}-\frac{\rho_{M} \phi^{M-1}}{M(M-1) ! \rho_{M-1}^{2} \prod_{i=1, i \neq M-1}^{M-2} \rho_{i}}-\lambda\left(\frac{\phi^{M}}{M ! \rho_{M-1}^{2} \prod_{i=1, i \neq M-1}^{M} \rho_{i}}\right)=0 . \\
\rho_{M-2} & =\sqrt{\frac{\rho_{M-1} \phi}{M-2}+\frac{\rho_{M} \phi^{2}}{\rho_{M-1}(M-1)(M-2)}+\frac{\lambda \phi^{3}}{\rho_{M} \rho_{M-1}(M-1)(M-2)}}
\end{aligned}
$$

Now, we cast the following power allocation problem:

$$
\begin{array}{ll}
\operatorname{minimize} & \rho_{\text {avg }} \\
\text { subject to } & 0 \leqslant \rho_{m}, 1 \leqslant m \leqslant M \\
& E_{M}=\epsilon
\end{array}
$$

where $\epsilon$ is any target outage probability. Notice that in (10) the optimization problem is non-linear and the feasible set is compact. Therefore, it is possible to find a global optimal solution [18]. In this paper, we obtain the optimal solution for the convex problem (10) by utilizing the Karush-Kuhn-Tucker (KKT) conditions.

We start our solution by writing the Lagrangian function for problem (10), which is

$$
\mathcal{L}\left(\rho_{m}, \mu_{m}, \lambda\right)=\frac{1}{M} \sum_{m=1}^{M} \rho_{m} E_{m-1}+\sum_{m=1}^{M} \mu_{m} \rho_{m}+\lambda\left(E_{M}-\epsilon\right),
$$

where $\mu_{m}$ for $m=1, \ldots, M$ is the inequality Lagrange multiplier and $\lambda$ is the equality Lagrange multiplier. Next, we write the KKT conditions as follows:

C1 $\frac{\partial \mathcal{L}}{\partial \rho_{m}}=0, m=1, \ldots, M$,

C2 $\mu_{m} \geqslant 0, m=1, \ldots, M$,

C3 $\mu_{m} \rho_{m}=0, m=1, \ldots, M$,

C4 $E_{M}-\epsilon=0$.

We start our solution from $\mathbf{C 1}$, and compute the derivative of the Lagrange function with respect to the power term of the $m^{\text {th }}$ round $\rho_{m}$ as shown in (12).

In each transmission round we require a certain amount of power to convey the information. This implies that, the transmit power at $m^{\text {th }}$ CC-HARQ round $\rho_{m}>0$ for $m=1, \ldots, M$. Thus, from the complementary slackness condition (C3) we notice that $\mu_{m}=0$ for $m=1, \ldots, M$. Now, using (12) and $\mu_{M}=0$, we write $\mathbf{C 3}$ for $m=M$ as in (13). After some manipulations of (13) we obtain the transmit power at the $M^{\text {th }}$ CC-HARQ round as

$$
\rho_{M}=\sqrt{\frac{M \lambda \phi}{M}}=\sqrt{\lambda \phi}
$$

Similarly, substituting $m=M-1$ in (12) and using $\mu_{M-1}=0$, we can rewrite $\mathbf{C 1}$ for $m=M-1$ as in (14).

Mathematical simplification of (14) leads to

$$
\rho_{M-1}=\sqrt{\frac{\rho_{M} \phi}{M-1}+\frac{\lambda \phi^{2}}{\rho_{M}(M-1)}} .
$$


Following a similar approach, we obtain the following relationship for the case of $m=M-2$ as in (15). We can continue this procedure for all $m \in\{1, \ldots, M\}$ and the results can be summarized as:

$$
\begin{aligned}
\rho_{M} & =f(\lambda) \\
\rho_{M-1} & =f\left(\lambda, \rho_{M}\right) \\
\rho_{M-2} & =f\left(\lambda, \rho_{M}, \rho_{M-1}\right) \\
\vdots & \\
\rho_{1} & =f\left(\lambda, \rho_{M}, \ldots, \rho_{3}, \rho_{2}\right)
\end{aligned}
$$

By utilizing a method that is similar to the backward substitution approach [19, App. C.2], we can obtain a relationship between the power terms $\rho_{m}$ as follows: first, by substituting $\lambda \phi=\rho_{M}^{2}$ (see (16) in (17)), we compute $\rho_{M-1}=\sqrt{\frac{2 \phi \rho_{M}}{M-1}}$. Next, $\rho_{M-2}$ is evaluated by substituting $\sqrt{\lambda \phi}=\rho_{M}$ and $\sqrt{\frac{2 \phi \rho_{M}}{M-1}}=\rho_{M-1}$ in (15). By continuing this procedure we can express the optimal transmit power in the $m^{\text {th }}$ round as

$$
\rho_{m}=\sqrt{\frac{2 \phi \rho_{m+1}}{m}}, 1 \leqslant m<M .
$$

Since $\rho_{M}$ is a function of $\lambda$ (see (16)) and using (22), it is obvious that each $\rho_{m}$ will also be a function of $\lambda$. Thus, all that remains is to compute the equality Lagrange multiplier $\lambda$. For this purpose, we utilize the last KKT condition, C4. First, we write $\rho_{m}$ in (9) as

$$
\rho_{m}=\frac{2^{a} \phi^{b} \lambda^{c}}{\sqrt{m}},
$$

where $a=2-2^{-(M-m-1)}, b=2-2^{-(M-m)}$ and $c=2^{-(M-m)}$. Then, we solve for $\lambda$ by equating $E_{M}$ to the outage target $\epsilon$.

\section{NUMERICAL ANALYSIS}

In Section IV, we propose a general framework for power allocation of $M$ transmission rounds. However, since URC systems are delay sensitive, we focus our analysis on a limited number of transmissions. Analytically, we evaluate the case of having a maximum of $M=2$ transmissions. For larger values of $M$, we resort to numerical methods. Moreover, when $M=2$, the optimization problem (10) simplifies to

$$
\begin{array}{ll}
\operatorname{minimize} & \frac{1}{2}\left(\rho_{1}+\rho_{2} \epsilon_{1}\right) \\
\text { subject to } & \frac{\phi^{2}}{2 \rho_{1} \rho_{2}}=\epsilon
\end{array}
$$

To solve problem (24) we can utilize the procedure described in Section IV. First, by using (16) and (22) we obtain the power terms as functions of $\lambda$. Then, we substitute these expressions into $\mathbf{C} \mathbf{4}$ and solving for $\lambda$ we attain $\lambda=\frac{\phi}{2 \epsilon} \sqrt[3]{\frac{1}{2 \epsilon}}$. Finally, the power terms are $\rho_{1}=\phi \sqrt[3]{\frac{1}{\epsilon}}$ and $\rho_{2}=\frac{\phi}{2 \epsilon} \sqrt[3]{\epsilon}$. When $M=2$ transmissions, we can employ the following approach to find the optimal power allocation. First, rewrite the equality constraint as $\rho_{2}=\frac{\phi^{2}}{2 \rho_{1} \epsilon}$ and substitute $\rho_{2}$ into the objective function of (24). This will result in an unconstrained optimization problem with variable $\rho_{1}$. Next, $\rho_{1}$ is calculated by setting the first derivative of the new objective function to zero as: $1-\frac{\phi^{3}}{\epsilon \rho_{1}^{3}}=0$; whose solution renders $\rho_{1}=\phi \sqrt[3]{\frac{1}{\epsilon}}$. Notice that this result matches the one obtained by using the procedure described in Section IV. Then, after substituting the first power term equation in the rewritten equality constraint we compute $\rho_{2}=\frac{\phi}{2 \epsilon} \sqrt[3]{\epsilon}$.

Fig. 2 we illustrates the power $\rho_{m}$ that needs to be transmitted in each CC-HARQ round to achieve a certain outage target $\epsilon$. First, we notice that both power terms are lower than the one shot transmission. Furthermore, we observe that if the first transmission is successful, then the power gains would be very large. For example, to operate in the ultra reliable region $\left(\epsilon \leqslant 10^{-3}\right)$, we can save more than $20 \mathrm{~dB}$ of power with respect to the one shot transmission. Also, notice that in the ultra reliable region, the first power term is lower than the second power term. This implies that, if the first transmission is unsuccessful, then a transmission with higher power takes place. Note that our goal is to achieve a target outage probability by spending as less power as possible. Since there is no delay limitation, we transmit first with low power. If the transmission encounters good channel conditions, then it will be successful, thus resulting in large power gains. Otherwise, we retry until succeed or the maximum allowed number of retransmissions is reached.

The behavior of the power terms as a function of the number of channel for the case when $M=2$ is illustrated in Fig. 3. To obtain the figure, we set the number of information bits $K \in\{100,200\}$ and $\epsilon=10^{-3}$. Notice that both power terms 


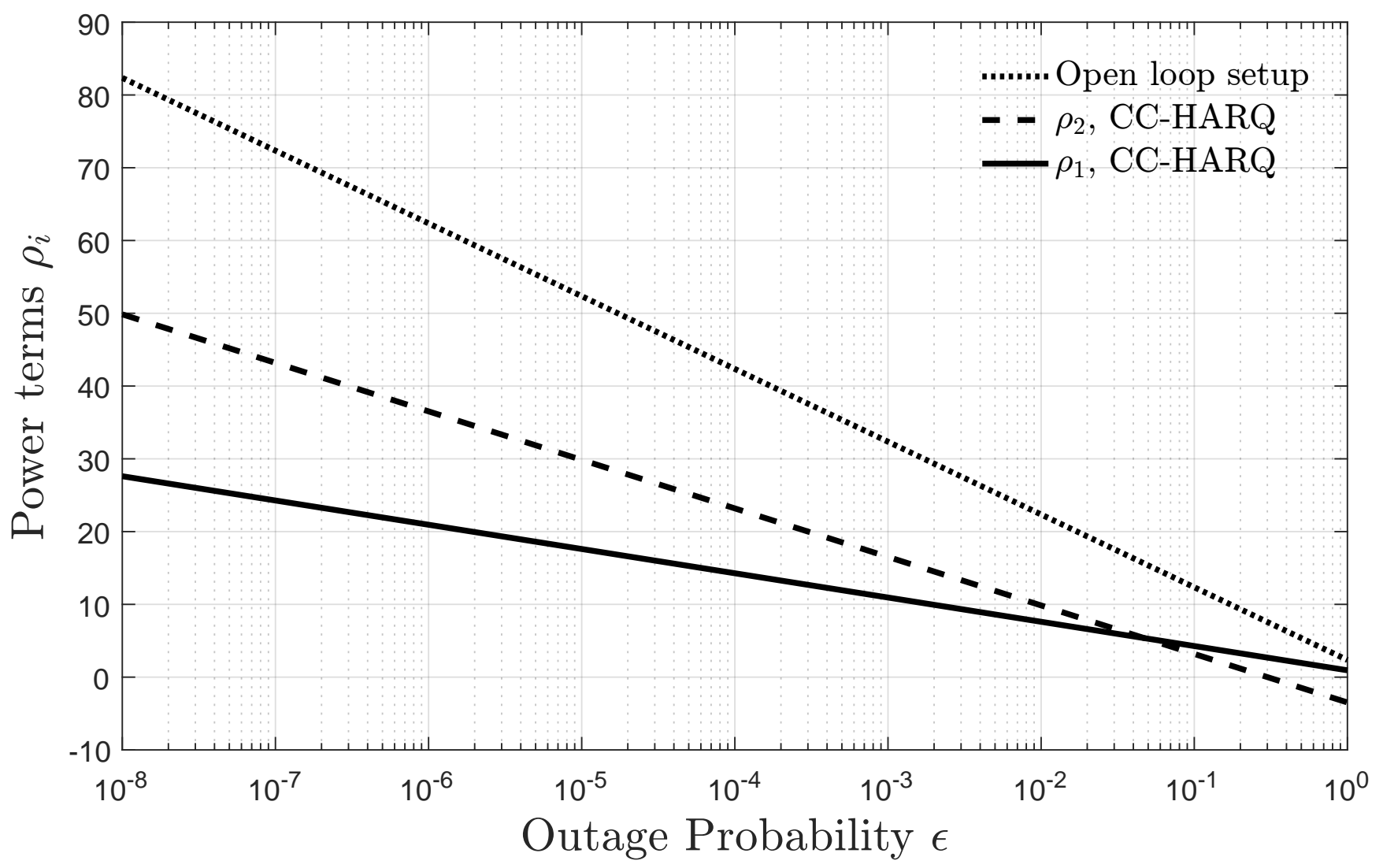

Fig. 2. Transmit power in each CC-HARQ round to achieve a target outage probability for rate $R=1 \mathrm{ncpu}$.

decrease as the number of channel uses increases. Secondly, we observe that when the coding rate increases, to maintain the same outage probability, the transmit power of each CC-HARQ round needs to be higher.

Finally, in Fig. 4 we analyze numerically the average and maximum power consumption of the proposed scheme for the case of $M=2$ and $M=3$ transmissions. To obtain the plot, we fixed $R=1 \mathrm{ncpu}$ and computed $\rho_{\text {avg }}$ as in (8). To compute the maximum power expenditure of the scheme, we assume that we utilize the maximum number of transmissions to convey a certain packet. Our results, show that the larger the number of transmissions is, the lower the power consumption will be. This happens due to the fact that we have higher diversity and combining gains. Furthermore, notice that the average gains attained with respect to the one shot transmission are quite high. Moreover, even in the worst case, we still save power. Specifically, to achieve $\epsilon=10^{-3}$, we save approximately $5 \mathrm{~dB}$ when $M=2$ and $10 \mathrm{~dB}$ when $M=3$.

\section{CONCLUSIONS AND FUTURE WORK}

In this paper, we have presented an optimal power allocation scheme for the CC-ARQ protocol. The proposed scheme, enables operation in the ultra reliable region, with minimal power expenditure. We have shown that we can attain large average and sum power gains. As future work, we intend to analyze the performance more complex retransmission protocols in the finite block-length regime. Furthermore, it would be of high interest to evaluate how the optimal scheme changes when considering feedback delay constraints.

\section{ACKNOWLEDGMENTS}

This work has been partially supported by Finnish Funding Agency for Technology and Innovation (Tekes), Huawei Technologies, Nokia and Anite Telecoms, and Academy of Finland (under Grant no. 307492).

\section{REFERENCES}

[1] P. Popovski, "Ultra-reliable communication in 5G wireless systems," in International Conference on 5 G for Ubiquitous Connectivity, Nov 2014.

[2] Ericsson, "Cellular networks for massive IoT," Ericsson white paper, January, 2016.

[3] Nokia Networks, "5G for mission critical communication: Achieve ultra-reliability and virtual zero latency," Nokia networks white paper, 2016.

[4] O. N. C. Yilmaz, Y. P. E. Wang, N. A. Johansson, N. Brahmi, S. A. Ashraf, and J. Sachs, "Analysis of ultra-reliable and low-latency 5g communication for a factory automation use case," in 2015 IEEE International Conference on Communication Workshop (ICCW), June 2015.

[5] H. Shariatmadari, S. Iraji, and R. Jantti, "Analysis of transmission methods for ultra-reliable communications," in 2015 IEEE $26 t h$ Annual International Symposium on Personal, Indoor, and Mobile Radio Communications (PIMRC), Aug 2015. 


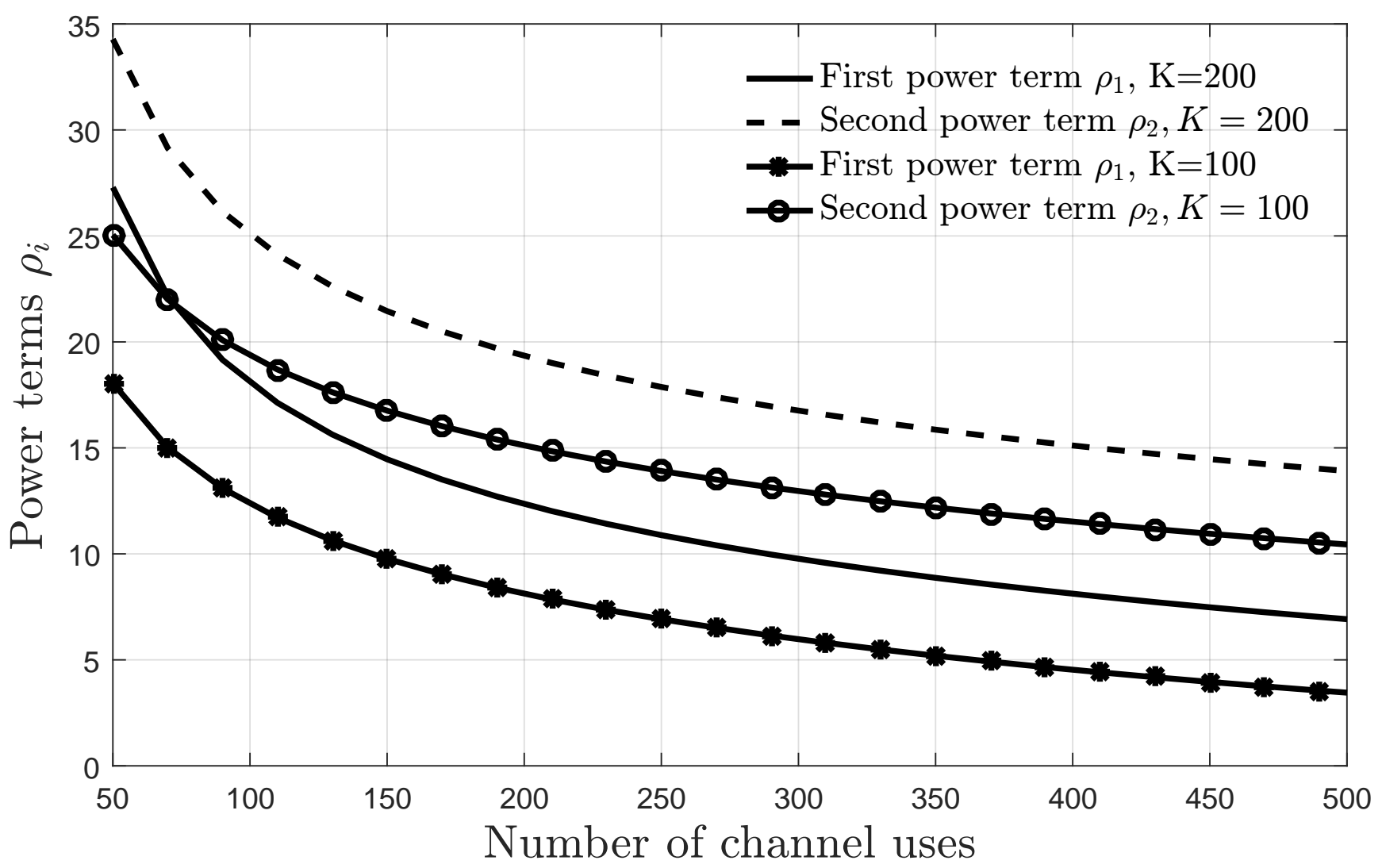

Fig. 3. Power terms as a function of the number of channel uses for different number of information bits.

[6] M. Simsek, A. Aijaz, M. Dohler, J. Sachs, and G. Fettweis, "5G-enabled tactile internet," IEEE Journal on Selected Areas in Communications, March 2016.

[7] A. Goldsmith, Wireless Communications. Cambridge, UK: Cambridge University Press, 2005.

[8] S. H. Kim, T. V. K. Chaitanya, and T. Le-Ngoc, "HARQ with chase-combining (HARQ-CC) for uplink transmission in large-antenna-array multicell systems," in 2016 IEEE 83rd Vehicular Technology Conference (VTC Spring), May 2016.

[9] G. Durisi, T. Koch, and P. Popovski, "Toward massive, ultra-reliable, and low-latency wireless communication with short packets," Proceedings of the IEEE, Sept 2016.

[10] E. Dosti, U. L. Wijewardhana, H. Alves, and M. Latva-aho, "Ultra reliable communication via optimum power allocation for Type-I ARQ in finite Block-Length," in IEEE ICC 2017 Wireless Communications Symposium (ICC'17 WCS), Paris, France, May 2017, pp. 5019-5024.

[11] S. H. Kim, D. K. Sung, and T. Le-Ngoc, "Performance analysis of incremental redundancy type hybrid ARQ for finite-length packets in AWGN channel," in IEEE GLOBECOM, 2013, pp. 2063-2068.

[12] R. Devassy, G. Durisi, P. Popovski, and E. G. Strom, "Finite-blocklength analysis of the ARQ-protocol throughput over the Gaussian collision channel," IEEE ISCCSP, pp. 173-177, 2014

[13] B. Makki, T. Svensson, and M. Zorzi, "Green communication via Type-I ARQ: Finite block-length analysis," in IEEE GLOBECOM, 2014.

[14] Y. Polyanskiy, H. V. Poor, and S. Verdu, "Channel coding rate in the finite blocklength regime," IEEE Transactions on Information Theory, vol. 56, no. 5, May 2010.

[15] W. Yang, G. Durisi, T. Koch, and Y. Polyanskiy, "Quasi-static multiple-antenna fading channels at finite blocklength," IEEE Transactions on Information Theory, vol. 60, no. 7, pp. 4232-4265, July 2014.

[16] T. V. K. Chaitanya and E. G. Larsson, "Optimal power allocation for hybrid arq with chase combining in i.i.d. rayleigh fading channels," IEEE Transactions on Communications, May 2013.

[17] J. N. Laneman, "Limiting analysis of outage probabilities for diversity schemes in fading channels," in Global Telecommunications Conference, 2003. GLOBECOM '03. IEEE, Dec 2003.

[18] T. V. K. Chaitanya and E. G. Larsson, "Outage-optimal power allocation for hybrid ARQ with incremental redundancy," IEEE Transactions on Wireless Communications, vol. 10, no. 7, July 2011.

[19] S. Boyd and L. Vandenberghe, Convex Optimization. Cambridge, UK: Cambridge University Press, 2004. 


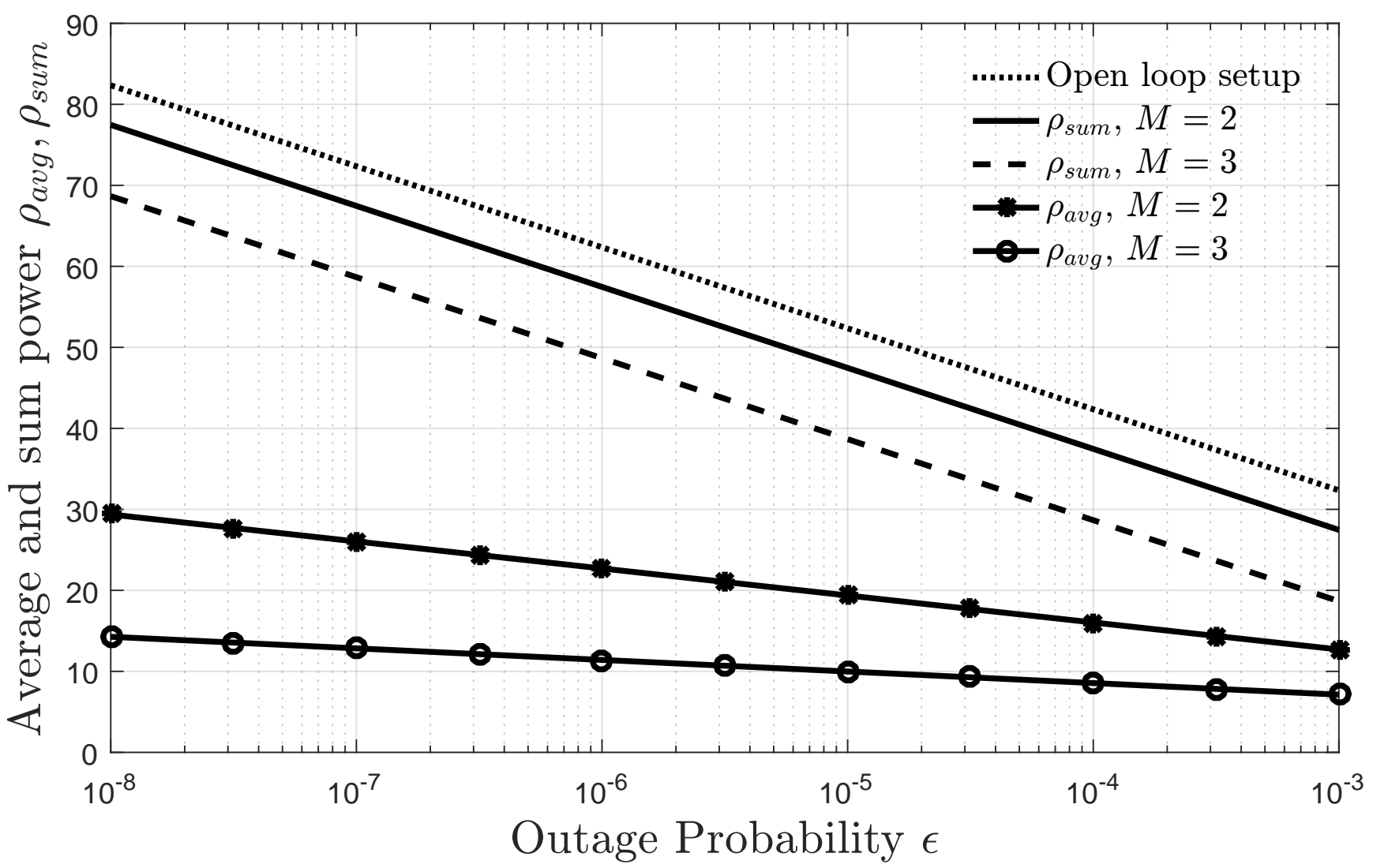

Fig. 4. Average and maximum power gains attained by the deployment of the proposed power allocation scheme. 